\title{
A existência de alterações neurofisiológicas pode auxiliar na compreensão do papel da hipotonia no desenvolvimento motor dos indivíduos com síndrome de Down?
}

\author{
Existence of neurophysiologic changes can assist in understanding the role of bypotonia \\ in motor development of subjects with Down syndrome?
}

João Carlos Ferrari Corrêa ${ }^{1}$, Adriano Rodrigues de Oliveira ${ }^{2}$, Cláudia Santos Oliveira ${ }^{1}$, Fernanda Ishida Corrêa ${ }^{1}$

Estudo desenvolvido no Laboratório de Biodinâmica do Movimento Humano da Universidade Nove de Julho (Uninove) - São Paulo (SP), Brasil.

${ }^{1} \mathrm{PhD}$; Professores do Programa de Mestrado e Doutorado em Ciências da Reabilitação da Uninove - São Paulo (SP), Brasil.

${ }^{2}$ MSc; Aluno do Programa de Mestrado em Ciências da Reabilitação da Uninove São Paulo (SP), Brasil.

\section{ENDEREÇO PARA CORRESPONDÊNCIA João Carlos Ferrari Corrêa - Av. Francisco Matarazzo, 612 - Água Branca - CEP: 05001-100 - São Paulo (SP), Brasil - E-mail: jcorrea@pq.cnpq.br \\ APRESENTAÇÃO maio 2011}

\section{ACEITO PARA PUBLICAÇÃO} out. 2011

\section{FONTE DE FINANCIAMENTO}

Este estudo foi suportado pela FAPESP (processo n²007/06078-0)

\section{CONFLITO DE INTERESSE} nada a declarar

Aprovado pelo Comitê de Ética e Pesquisa $\mathrm{n}^{\circ}$ 122488/07
RESUMO: A proposta deste estudo foi analisar e verificar a existência de alterações neurofisiológicas nos indivíduos com síndrome de Down, como a hiporreflexia, estática e dinâmica, que, se presente, pode ocasionar prejuízo no controle sensóriomotor dos músculos, e consequentemente a hipotonia, contribuindo para alterações no desenvolvimento motor. O sinal eletromiográfico (EMG), início e término do sinal eletromiográfico, foi coletado de 24 voluntários com diagnóstico de síndrome de Down e 25 voluntários como grupo controle, durante 2 situações distintas: período de latência dinâmico e teste de reflexo monossináptico (estático) patelar e aquileu. Em relação aos aspectos neurofisiológicos, observou-se que, para todas as variáveis analisadas, houve diferença estatisticamente significante $(p<0,05)$ entre os grupos. A existência de alterações neurofisiológicas (retardo do período de latência) comprovadas neste estudo, oriundas de um prejuízo no controle sensório-motor, as quais podem ser responsáveis pela hipotonia muscular, mostrou-se como um dos vários possíveis fatores determinantes pelas alterações no desenvolvimento motor dessas crianças, tornando-se importante devido às alterações neuromusculares que podem interferir nas atividades funcionais.

Descritores: síndrome de Down; neurofisiologia; função sensorial.

ABSTRACT: The purpose of this study was to analyze and verify the existence of neurophysiological changes in individuals with Down syndrome, such as hyporeflexia, static and dynamic, which, if present, may cause impairment in sensory-motor control of muscles, and consequently hypotonia, contributing to changes in motor development. The electromyographic signal (EMG), start and termination electromyographic signal, was collected from 24 volunteers with a diagnosis of Down syndrome and 25 volunteers as controls for 2 different situations: the dynamic latency period and monosynaptic reflex (static) patellar and Achilles. For neurophysiological aspects, it was observed that for all variables analyzed, statistical difference $(p<0.05)$ between groups. The existence of neurophysiological changes (delay of the latency period) demonstrated in this study originated from an impairment in sensory-motor control, which may account for the decreased muscle tone, presents as one of several possible factors for the changes in motor development of children, which shows important due to neuromuscular disorders that can interfere in functional activities.

KEYWoRDs: Down syndrome; neurophysiology; sensation. 


\section{INTRODUCÃ̃}

A síndrome de Down (SD) é uma alteração cromossômica, acompanhada de múltiplas alterações congênitas, que podem gerar diversas complicações no desenvolvimento neurofisiológico e motor dos indivíduos portadores dessa síndrome, tais como hipotonia e disfunções motoras dinâmicas, dentre as quais destacamos o aumento do tempo de reação, movimentos lentos, atraso no desenvolvimento motor, déficits de equilíbrio postural e de co-contração de musculatura agonista e antagonista ${ }^{1,2}$. Aspectos como hipotonia, fraqueza muscular e hipoplasia cerebelar vêm sendo sugeridos como causa do atraso no desenvolvimento motor (aquisição dos marcos motores) para a criança com $\mathrm{SD}^{3}$.

Estudos pioneiros (por exemplo, $\mathrm{Carr}^{4}$ e Melyn e White ${ }^{5}$ ) têm demonstrado que crianças com SD normalmente não adquirem habilidades motoras com a mesma taxa de desenvolvimento típica de seus pares. Segundo estudo realizado por Pueschel ${ }^{6}$, pode-se observar os seguintes marcos em relação ao desenvolvimento motor na criança com SD: sentar de forma independemente entre 6 e 28 meses; ficar de pé entre 11 e 42 meses; e deambular entre 12 e 65 meses.

Apesar dessas evidências, as crianças com SD parecem seguir a mesma sequência do desenvolvimento motor de lactentes saudáveis ${ }^{7}$, embora diferenças qualitativas ou padrões de movimento atípicos, a fim de manter a estabilidade postural, sejam muitas vezes relatados. Por exemplo, muitas vezes essas crianças sentam-se com pernas afastadas, têm a marcha com base larga e passam de prono para sentado estendendo as pernas e empurrando com as mãos ${ }^{8}$. Posturas incomuns podem ser também relacionadas à presença de hipotonia, frequentemente associada com a síndrome.

A hipotonia muscular está certamente relacionada com a reduzida força em crianças e adolescentes, mesmo se a exata influência da hipotonia no desenvolvimento motor não for determinada. A hipotonia associada com a SD manifestase ainda na fase intra-útero e mantém-se após o nascimento de uma forma moderada, podendo ser acompanhada de uma hiporreflexia ${ }^{9}$. Essa hipotonia é uma das principais causas das disfunções motoras e, consequentemente, da alteração no controle postural das crianças com síndrome de Down ${ }^{10}$, a qual depende de uma integração contínua entre o sistema sensorial e o motor ${ }^{11}$.

O estudo do reflexo muscular, que tem por função garantir o tônus muscu$\operatorname{lar}^{12}$ e que é resultante da estimulação do fuso neuromuscular (sistema sensorial) causando contração reflexa do músculo (sistema motor), pode auxiliar na compreensão do papel da hipotonia no desenvolvimento motor (atraso na aquisição dos marcos motores) das crianças portadoras de $\mathrm{SD}^{13}$, principalmente se for detectada a diminuição da velocidade de condução do potencial de ação, de forma crônica por meio da análise dos reflexos monossináptico (estático) patelar e aquileu, além do reflexo dinâmico, sendo eles propostos neste estudo pela eletromiográfica [início e término do sinal eletromiográfico (EMG)].

Embora a hipotonia seja uma das mais frequentes características musculares em crianças com SD, seu papel no desenvolvimento motor está longe de ser determinado ${ }^{13,14}$. Sendo assim, a proposta deste estudo foi analisar e verificar a existência de alterações neurofisiológicas nos indivíduos com SD, como a hiporreflexia, estática e dinâmica, que, se presente, pode ocasionar prejuízo no controle sensório-motor dos músculos, e consequentemente a hipotonia, contribuindo para alterações no desenvolvimento motor, sendo esta verificada pela eletromiografia, ferramenta pouco utilizada para o estudo desse sintoma (hipotonia) na literatura.

\section{MATERIAL E MÉTODOS}

Este estudo é de caráter transversal controlado. Os objetivos e procedimentos realizados foram previamente explicados aos voluntários, obtendo-se o termo de consentimento livre e esclarecido deles para participação da pesquisa clínica, conforme aprovado pelo Comitê de Ética em Pesquisa (processo 122488/07).

\section{Sujeitos}

Após o cálculo estatístico do tamanho da amostra, tendo-se uma alteração neurofisiológica de 4\% após o agravamento do período de latência reflexo como variável-alvo, e admitindo-se erro $\alpha$ de $5 \%$ e erro $\beta$ de $20 \%$, foram triados 24 voluntários com diagnóstico de síndrome de Down e 25 voluntários como grupo controle (controle saudável), todos eles apresentando idade entre 16 e 25 anos, de ambos os sexos, pensamento cognitivo suficiente para compreensão dos exercícios propostos, capacidade de manter-se em ortostatismo sem apoio e não apresentar nenhuma doença ortopédica, alteração vestibular, auditiva ou visual, não fazer uso de medicação que alterasse a transmissão nervosa, não possuir neuropatia periférica nem tão pouco problemas respiratórios que alterassem a relação ventilação-perfusão.

Procedimento

\section{Testes para avaliação dos aspectos neurofisiológicos}

Foi utilizado um eletromiógrafo*, módulo condicionador de sinais e quatro pares de eletrodos de superfície. Os componentes do sistema de aquisição de sinais, eletrodos, foram conectados a um módulo condicionador de sinais, onde os sinais analógicos, já amplificados em anteriormente em dez vezes, foram amplificados novamente com um ganho de cem vezes, totalizando, portanto, um ganho final de mil, e filtrados com filtro passa-banda de 10 a $500 \mathrm{~Hz}$.

Os 4 pares de eletrodos de superfície do tipo ativo, bipolar, diferencial e razão do modo comum de rejeição igual a $80 \mathrm{~dB}$ foram colocados sobre os músculos reto femoral (RF), fibular longo $(\mathrm{FL})$, fibular curto (FC) e sóleo (SO), do lado dominante, conforme recomendações do Projeto SENIAM (Surface Electromyography for the Non-Invasive Assessment of Muscles) ${ }^{15}$.

O valor de RMS (root mean square) do sinal eletromiográfico foi coletado durante duas situações distintas: período de latência dinâmico e reflexo monossináptico (estático). Para a marcação do início e término do sinal EMG, utilizou-se duas vezes o desvio padrão médio do sinal de referência:

1. Período de latência dinâmico: um traçado médio do período de latência (início e cessação) para a resposta muscular dos músculos fibular curto e longo, analisados neste estudo após

EMG System do Brasil 
incitado um desequilíbrio em um banco de madeira, por mecanismo próprio (trava que, após seu desarme, causa uma inclinação de $30^{\circ}$ na base de apoio do voluntário, causando um desequilíbrio inesperado e tendo como resposta a contração muscular reflexa), adaptado com um switch em sua área de desarme do mecanismo, sendo representativo da atividade funcional dos músculos de cada voluntário. Esse banco de madeira possui $60 \times 30 \times 35 \mathrm{~cm}$ (largura, comprimento e altura), sendo dividido fisicamente ao meio, tendo, portanto, 2 superfícies distintas de $30 \times 30 \mathrm{~cm}$. Uma dessas superfícies inclina-se em um ângulo de $30^{\circ}$, causando um desequilíbrio em inversão.

2. Teste de reflexo monossináptico: foi realizado por meio do martelo de exame neurológico adaptado com um switch em sua área de percussão.

- Patelar: o reflexo patelar é um reflexo profundo, mediado por nervos oriundos da raiz de L2, L3 e L4. Clinicamente é considerado como um reflexo de $\mathrm{L} 4$. O paciente deve sentar-se com as pernas pendentes, e, em seguida, percute-se o tendão patelar com um golpe curto e rápido sobre o ligamento patelar, observando a extensão do joelho ${ }^{16}$.

- Aquileu: também classificado como reflexo profundo, mediado pelos músculos gastrocnêmio e sóleo, referente ao nível neurológico S1. Com as pernas pendentes, o tendão de Aquiles deve ser levemente tensionado por meio de uma discreta dorsiflexão do pé. Para testá-lo, deve-se percurtir o tendão, induzindo uma flexão plantar involuntária ${ }^{16}$.

\section{Análise estatística}

Para testar a normalidade ou não das variáveis, foi utilizado o teste KolmogorovSmirnov (KS). Como as variáveis estudadas apresentaram distribuição normal, para realizar a análise estatística dos resultados foi utilizado o teste $t$ de Student para comparar a média dos dados entre os dois grupos.
RESULTADOS

A amostra inicial foi constituída de 33 pacientes, dos quais 9 foram excluídos, totalizando 24 pacientes (Tabela 1). Quatro pacientes foram excluídos por apresentar desvios posturais associados à síndrome de Down, um paciente foi excluído por apresentar alteração auditiva, e quatro pacientes foram excluídos por faltarem nas datas agendadas para avaliação e coleta dos dados.

Como grupo controle, utilizamos um grupo de voluntários saudáveis (Tabela 1), que se mostraram semelhantes, sem diferença estatisticamente significante, em relação à idade, peso, altura e IMC $(p>0,05)$.

\section{Avaliação dos aspectos neurofisiológicos}

Em relação aos aspectos neurofisiológicos, observou-se que, para todas variáveis analisadas, houve diferença estatisticamente significante $(p<0,05)$ entre os grupos (Tabela 2).

Tabela 1. Dados médios e respectivos desvios padrão das características demográficas dos voluntários portadores de síndrome de Down (SD) e do grupo controle (GC). Exceção feita ao gênero dos voluntários, apresentados em número absoluto

\begin{tabular}{lcc}
\hline & $\mathrm{GC}$ & $\mathrm{SD}$ \\
& $\mathrm{n}=25$ & $\mathrm{n}=24$ \\
\hline Idade (anos) & $20,23( \pm 2,40)$ & $19,64( \pm 3,10)$ \\
Massa $(\mathrm{kg})$ & $63,45( \pm 5,43)$ & $65,07( \pm 4,34)$ \\
Altura $(\mathrm{m})$ & $1.65( \pm 0,23)$ & $1.60( \pm 0,12)$ \\
Gênero & $7 \mathrm{~F}-8 \mathrm{M}$ & $8 \mathrm{~F}-6 \mathrm{M}$ \\
$\mathrm{IMC}\left(\mathrm{kg} / \mathrm{m}^{2}\right)$ & $22,62 \pm 3,79$ & $28,08 \pm 6,00$ \\
\hline
\end{tabular}

Tabela 2. Dados médios e respectivos desvios padrão referentes ao período de latência dos reflexos tendinoso e dinâmico - inversão de tornozelo (avaliações neurofisiológicas) dos voluntários portadores de síndrome de Down (SD) e do grupo controle (GC)

\begin{tabular}{lccc}
\hline & $\begin{array}{c}\mathrm{GC} \\
\mathrm{n}=25\end{array}$ & $\begin{array}{c}\mathrm{SD} \\
\mathrm{n}=24\end{array}$ & Valor $\mathrm{p}$ \\
\hline $\begin{array}{l}\text { Latência RTP }(\mathrm{ms}) \text { - músculo reto } \\
\text { femoral }\end{array}$ & $24,81( \pm 8,62)$ & $31,17( \pm 4,75)$ & 0,020 \\
Latência RTA $(\mathrm{ms})$ - músculo sóleo & $28,29( \pm 12,31)$ & $40,92( \pm 4,14)$ & 0,018 \\
$\begin{array}{l}\text { Reflexo dinâmico (ms) - músculos } \\
\text { fibular longo e fibular curto }\end{array}$ & $68,72( \pm 4,92)$ & $94,92( \pm 2,78)$ & 0,034 \\
\hline
\end{tabular}

RTP: reflexo tendinoso patelar; RTA: reflexo tendinoso aquileu; ms: milissegundos 
Frijins e colaboradores ${ }^{18}$ realizaram um estudo para pesquisar os valores normais dos reflexos patelar e aquileu em 120 voluntários com idade média de 36,9 sem problemas neurológicos que interferissem nas respostas reflexas. Para testar o reflexo aquileu, o paciente era posicionado em decúbito ventral com os pés para fora da maca, em que foram colocados eletrodos de $8 \mathrm{~mm}$ de diâmetro na região do músculo sóleo. $\mathrm{O}$ eletrodo de referência ficava $5 \mathrm{~cm}$ abaixo do nervo tibial sobre a fossa poplítea, com frequência de estímulo de $0,2 \mathrm{~Hz}$ e duração de $0,5 \mathrm{~ms}$, sendo gravado o período de latência do reflexo H. Já o reflexo patelar era testado após o reflexo aquileu, em que o paciente ficava em sedestação com aproximadamente $135^{\circ}$ de flexão de joelhos e com o eletrodo posicionado no ventre do músculo reto femoral. Os resultados encontrados foram um período de latência de $21,0 \pm 1,5$ e $35,2 \pm 2,6 \mathrm{~ms}$ para o refelexo tendinoso patelar e aquileu, respectivamente.

Nossos resultados apontam para um acerto da escolha da metodologia utilizada, uma vez que para o grupo controle (GC) os dados são semelhantes aos estudos apresentados além de apontar a existência de uma alteração significativa de condução neurofisiológica, conforme apresentado por Kayacan e colaboradores $^{19}$, que pode significar algum marcador diagnóstico, funcional ou ainda prognóstico. Contudo, mais estudos são necessários no sentido de verificar uma correlação forte e positiva das variáveis aqui analisadas e de avançar na proposta sugerida.

O estudo do reflexo muscular pode auxiliar na compreensão do papel da hipotonia no desenvolvimento motor, principalmente ao se detectar a diminuição da velocidade de condução do potencial de ação por meio da eletromiografia.

Achamos que a diferença na resposta reflexa, encontrada neste estudo, pode estar relacionada a alterações sensoriais. O reflexo medular acontece por estímulo de receptores que estão presentes no interior do músculo (fuso neuromuscular e o complexo de Golgi). Esses receptores são estimulados pelo cumprimento e velocidade da contração muscular, e uma contração lenta pode estimular de forma ineficaz esses receptores.

Outra hipótese possível é a própria hipotonia presente nesses pacientes com $\mathrm{SD}$, ao fazer com que o músculo realize uma contração muscular mais lenta e/ou ineficaz, levando-o assim a um tempo de resposta maior. Pode-se definir tônus muscular como sendo o "estado de turgidez do músculo causado pela plasticidade muscular (consistência) e pelos reflexos de estiramentos". Já a hipotonia caracteriza-se, fisiologicamente, como uma diminuição segmental da excitabilidade do pool de motoneurônios, e como um mecanismo de reflexo de estiramento comprometido (hiporreflexia) ${ }^{14}$, consequência de um controle sensório-motor diminuído.

Já com relação à resposta reflexa dinâmica, também podemos notar uma diferença significante comparando os resultados entre os grupos (Tabela 2 ).

$\mathrm{Na}$ literatura, encontramos poucos trabalhos com a mesma metodologia empregada neste estudo. Fritschye Reynier ${ }^{20}$ estudaram a instabilidade lateral crônica submetidos à cirurgia de reforço do ligamento lateral, obtendo como resposta de latência reflexa dinâmica 118 ms. Löfarnberg, Kärrholm e Sundelin ${ }^{21}$ estudaram o tempo de resposta reflexa também em indivíduos com instabilidade ligamentar crônica do tornozelo (85 ms), em que o tempo de reação foi comparado com o do grupo controle (49 ms) e demonstrado um aumento significativo no tempo de reação dos músculos.

Em trabalhos de Schimidt e colaboradores $^{22}$, em que foi utilizada uma metodologia semelhante a nossa, foram analisados 120 voluntários saudáveis e jovens com idade média inferior a 30 anos, com o uso da plataforma móvel (instável) e eletromiografia nos músculos fibular longo e curto, tendo como resposta do período de reflexo dinâmico de 25 ms. Já no estudo de Kermorzek e colaboradores ${ }^{23}$, participaram 28 voluntários saudáveis, de 19 a 30 anos, em 2 condições: com brace e sem brace, sendo avaliada a inversão $\left(30^{\circ}\right)$ em 3 sub-condições: 10, 20 e 30 graus de flexão plantar. Entre todas as condições, com uma média de 51,20 ms na resposta reflexa dinâmica, nenhuma diferença estatisticamente significante foi observada.

A inversão de tornozelo pode ser utilizada como uma resposta reflexa para a manutenção do equilíbrio em uma atividade que cause a perturbação do tornozelo. Isso ocorre devido aos mecanorrecptores que estão presentes nos músculos e nos ligamentos dessa articulação.

A resposta de latência reflexa dinâmica em indivíduos portadores de SD é maior quando comparando com indivíduos saudáveis, podendo estar relacionada a uma alteração na resposta sensório-motora. Para que ocorra a resposta reflexa, é preciso ativar os mecanorrecptores que estão presentes nos músculos; e como esses pacientes apresentam uma alteração somatosensorial, essa ativação pode ocorrer de forma inadequada, causando assim um potencial de ação mais baixo.

Essa diminuição da resposta de latência reflexa dinâmica também pode estar relacionada à hipotonia presente nesses indivíduos ${ }^{24}$, em virtude de não ser possível regular o controle muscular para se manter em equilíbrio.

\section{CONCLUSÃO}

Verificamos com este estudo e por meio da eletromiografia que, para os portadores da síndrome de Down, a existência de diminuição da velocidade de condução do potencial de ação, de forma crônica, pode ocasionar prejuízo no controle sensório-motor dos músculos e, consequentemente, a hipotonia desses indivíduos. Respostas reflexas são importantes para o controle sensório-motor, e sua avaliação e interpretação podem colaborar para mais investigações no âmbito reabilitativo desses indivíduos. 


\section{REFERENCIAS}

1. Galli M, Rigoldi C, Mainardi L, Tenore N, Onorati P, Albertini G. Postural control in patients with Down syndrome. Disabil Rehabil. 2008;30(17):1274-8.

2. Dierssen M, Ortiz-Abilia J, Arqué G, de Lagran MM, Fillat C. Pitfalls and hopes in Down syndrome therapeutic approaches: in the search for evidence-based treatments. Behav Genet. 2006;36(3):454-68.

3. Meneghetti CHZ, Blascovi-Assis SM, Deloroso FT, Rodrigues GM. Avaliação do equilíbrio estático de crianças e adolescentes com síndrome de Down. Rev Bras de Fisioter. 2009;13(3):230-5.

4. Carr, J. Mongolism: telling the parents. Dev Med Child Neurol. 1970;12(2):213-21.

5. Melyn MA, White DT. Mental and developmental milestones of noninstitutionalized Down's syndrome children. Pediatrics. 1973;52(4):542-5.

6. Pueschel SM. Clinical aspects of Down syndrome from infancy to adulthood. Am J Med Genet. 1990;7(suppl.):52-6.

7. Palisano RJ, Walter SD, Russell DJ, Rosenbaum PL, Gemus M, Galuppi BE, et al. Gross motor function of children with down syndrome: creation of motor growth curves. Arch Phys Med Rehabil. 2001;82(4):494-500.

8. Lydic JS, Steele C. Assessment of the quality of sitting and gait patterns in children with Down's syndrome. Phys Ther. 1979;59(12):1489-94.

9. Shumway-Cook A, Woollacott M. Dynamics of postural control in child with Down syndrome. Phys Ther. 1985;65(9):1315-22.

10. Mazzone L, Mugno D, Mazzone D. The General Movements in children with Down syndrome. Early Hum Dev. 2004;79(2):119-30.

11. Sasaki O, Usami S, Gagey PM, Martinerie J, Le Van QM, Arranz P. Role of visual input in nonlinear postural control system. Exp Brain Res. 2002;147(1):1-7.

12. Naznin V, Babul I, Kerns K, et al. Perceptual - Motor deficits in children with Down Syndrome: Implication for intervention. Down Syndrome Res. Prat.. 2006;10:74-8.

13. Vicari S. Motor development and neuropsychological patterns in persons with Down syndrome. Behav Genetics. 2006;36(3):355-64.
14. Haslan RHA. Neurological problems associated with Down syndrome. In Nadel L, Rosenthal D, editors. Down syndrome: living and learning in the community. New York: Wiley-Liss; 1995. p. 107-14.

15. Hermens HJ, Freriks B, Merletti R et al. Project SENIAM (Surface Electromyography for the Non-Invasive Assessment of Muscles). European Recommendations for Surface Electromyography, deliverable of the SENIAM project Disselhorst-Klug, Roessingh Research and Development b.v., 1999.

16. Hoppenfeld S. Propedêutica Ortopédica: coluna e extremidades. Rio de Janeiro: Atheneu, 1987. p. 200-1 e 241-3.

17. Feinberg J. EMG: Myths and Facts. HSS J. 2006;2(1):19-21.

18. Frijns CJ, Laman DM, van Duijn MA, van Duijn $\mathrm{H}$. Normal values of patellar and ankle tendon reflex latencies. Clin Neurol Neurosurg. 1997;99(1):31-6.

19. Kayacan O, Beder S, Deda G, Karnak D. Neurophysiological changes in COPD patients with chronic respiratory insufficiency. Acta Neurol Belg. 2001;101(3):160-5.

20. Fritschy D, Reynier JC. Plastic of the ligament for chronic lateral instability of the ankle. Int Orthop. 1988;12(3):239-47.

21. Löfvenberg R, Kärrholm J, Sundelin G. Proprioceptive reaction in the healthy and chrocically unstable ankle joint. Sportsveletz Sportschaden. 1996;10(4):79-83.

22. Schimidt R, Gergroü H, Friemert B, Herbst A, Claes L. The peroneal reaction time (PRT) - reference data in healthy sample. Foot Ankle Int. 2005;26(5):382-6.

23. Kermorzek T, Durall CJ, Friske A, Mussallem M. Ankle bracing, plantar-flexion angle, and ankle muscle latencies during inversion stress in healthy participants. J Athl Train. 2008;43(1):37-43.

24. Juanhua WD, Ulrich A, Julia L, et al. Strategy adoption and locomotor adjustment in obstacle clearance of newly walking toddlers with Down syndrome after different treadmill interventions. Exp Brain Res. 2008;186(2):261-72. 